\title{
Tendência das internações por condição sensível à atenção primária e fatores associados em Porto Alegre, RS, Brasil
}

\author{
Trends in hospitalization for primary care-sensitive conditions \\ and associated factors in Porto Alegre, Rio Grande do Sul, Brazil
}

\author{
Juvenal Soares Dias da Costa ${ }^{1}$ \\ Marcos Pascoal Pattussi ${ }^{1}$ \\ Tissiani Morimoto ${ }^{1}$ \\ Jocinei Santos de Arruda ${ }^{1}$ \\ Gabriela Rodrigues Bratkowski ${ }^{1}$ \\ Mariani Sopelsa ${ }^{1}$ \\ Janaina Soder Fritzen ${ }^{1}$ \\ Vaneza de Andrade da Fontoura do Canto ${ }^{1}$ \\ Maximiliano Chagas Marques ${ }^{1}$
}

${ }^{1}$ Programa de PósGraduação em Saúde Coletiva, Universidade do Vale do Rio dos Sinos. Av. Unisinos 950, Cristo Rei.

93.022-000 São Leopoldo RS Brasil.

episoares@terra.com.br

\begin{abstract}
An ecological study was conducted to analyze trends in hospitalization for primary care-sensitive conditions linking the results to the investments in health and coverage of the Family Health Strategy in Porto Alegre, between 1998 and 2012. The causes of hospitalization for primary care-sensitive conditions were based on the national list provided by the Ministry of Health. The data were obtained from the Hospital Information System of the Unified Health System (SUS). Standardized rates were created and investments increased by 27\%, though investments in primary care increased by $83 \%$. The expansion of coverage by the Family Health Strategy was almost fourfold, though it remained below the recommended values. There was no change in the trend of hospitalization for primary care-sensitive conditions. The analysis did not make it possible to establish if patients who were hospitalized for primary care-sensitive conditions had access to the Family Health Strategy or not, suggesting the need to incorporate data of place of origin in the information system. Studies using the Hospital Information System contribute to its enhancement, fomenting the assessment, management and design of health policies.
\end{abstract}

Key words Quality of healthcare, Primary healthcare, Hospitalization, Family health, Healthcare financing
Resumo Realizou-se estudo ecológico para analisar a tendência das internações por condições sensíveis à atenção primária relacionando-as com os investimentos financeiros em saúde e a cobertura da Estratégia Saúde da Família em Porto Alegre, entre 1998 e 2012. As causas de internações por condições sensíveis à atenção primária foram baseadas na lista nacional, conforme o Ministério da Saúde. Os dados foram retirados do Sistema de Informações Hospitalares do SUS. Foram construidas taxas padronizadas. Os investimentos financeiros aumentaram $27 \%$ e em atenção primária $83 \%$. A expansão da cobertura pela Estratégia Saúde da Família foi de cerca de quatro vezes, entretanto, continuou abaixo do preconizado. Não houve alteração na tendência das internações por condiçães sensíveis à atenção primária. A análise não permitiu distinguir se os pacientes que internaram por condições sensiveis à atenção primária tinham acesso à Estratégia Saúde da Família ou não, sugerindo a necessidade de se incorporar no sistema de informações dados sobre o local de origem. Estudos utilizando o Sistema de Informações Hospitalares contribuem para seu aprimoramento, propiciando a avaliação, a gestão e o delineamento de politicas de saúde.

Palavras-chave Qualidade da assistência à saúde, Atenção Primária à Saúde, Hospitalização, Saúde da família, Financiamento da assistência à saúde 


\section{Introdução}

O Brasil lançou em 1994 o Programa Saúde da Família, hoje transformado em Estratégia Saúde da Família (ESF), com o objetivo de mudança no modelo de atenção vigente. Essa estratégia surgiu para reorganizar a utilização dos níveis assistenciais de forma a racionalizar o atendimento, contribuindo para a melhora do acesso e da qualidade da Atenção Primária à Saúde (APS) ${ }^{1,2}$.

Para que a atenção primária forneça cuidados de saúde de qualidade, são necessários atributos como acesso facilitado, atenção efetiva e equânime, com custos compatíveis ${ }^{3}$. A Organização Mundial de Saúde apontou como principal proposta de modelo assistencial a atenção primária, tendo em vista, por exemplo, a melhoria dos indicadores de saúde e a maior eficiência aos gastos do setor ${ }^{4}$. Dentro deste contexto, torna-se necessário a avaliação permanente dos sistemas de saúde. Um dos indicadores de avaliação é denominado de Internações por Condições Sensíveis à Atenção Primária (ICSAP), o qual é baseado em um conjunto de doenças que, se tratadas de forma efetiva na atenção primária, reduzem o risco de internações hospitalares ${ }^{5}$. Este indicador foi desenvolvido na década de 1990 por Billings et al. ${ }^{6}$ e está consolidado na literatura, sendo utilizado em diversos estudos nacionais e internacionais $^{5,7-9}$.

O Ministério da Saúde brasileiro criou sua própria lista em 2008, levando em consideração a realidade do país e permitindo a comparabilidade entre os estudos nacionais. A utilização deste indicador possibilita aos gestores o aprimoramento do planejamento e da gestão dos serviços de saúde, uma vez que permite avaliar o desempenho da atenção primária ${ }^{1}$.

Contudo, os investimentos financeiros realizados em saúde no Brasil, nos três níveis de atenção, ainda são insuficientes. Destaca-se que há uma desigualdade em investimentos, tendo em vista a maior escassez de recursos, principalmente na atenção primária, devido a uma visão distorcida da sua complexidade, o que leva à desvalorização deste nível de atenção ${ }^{10-12}$. Segundo dados de 2008 a 2010 de um estudo realizado no interior de São Paulo, as ICSAP geraram um gasto de $\mathrm{R} \$ 30.370 .691,08$, o que representou $17 \%$ dos investimentos financeiros totais realizados em saúde ${ }^{13}$. Em outro estudo, Dias da Costa et al. ${ }^{14}$ encontraram uma redução do percentual de gastos com ICSAP em relação aos gastos totais no decorrer dos anos analisados, de 1995 a 2004, passando de $13,0 \%$ para $5,4 \%$.
Desta forma, o presente estudo teve por objetivo analisar a tendência das ICSAP relacionando -as com os investimentos financeiros em saúde e a cobertura da ESF no município de Porto Alegre, Rio Grande do Sul, no período de 1998 a 2012.

\section{Metodologia}

Trata-se de estudo ecológico para analisar a tendência das ICSAP no município de Porto Alegre, no período de 1998 a 2012.

Porto Alegre possui 1.409.351 habitantes de acordo com o Censo de 2010. As principais portas de acesso à atenção primária a saúde no município são 55 unidades básicas de saúde (UBS) e 88 unidades de saúde da família (USF). Oferece, ainda, atendimento em oito centros de saúde. Os atendimentos de urgência e emergência são ofertados em oito unidades mantidas pelo município e em 11 unidades conveniadas. Porto Alegre conta com quatro hospitais municipais e 21 conveniados, e é a principal referência do Estado.

Os dados secundários foram retirados do DATASUS (www.datasus.gov.br) por meio do Sistema de Informações Hospitalares do Sistema Único de Saúde (SIH/SUS). As informações populacionais foram obtidas por meio do Instituto Brasileiro de Geografia e Estatística (IBGE).

Foram elaboradas as taxas brutas para todas as causas de internação, excluindo-se as obstétricas. Também foram construídas taxas brutas de ICSAP. As causas de internações por condições sensíveis à atenção primária foram baseadas na lista nacional, conforme a Portaria do Ministério da Saúde n 221, de 17 de abril de 2008.

Os dados financeiros de Porto Alegre foram extraídos dos extratos de repasses disponíveis no Sistema de Informações sobre Orçamentos Públicos em Saúde (www.saude.gov.br/siops). Os indicadores foram elaborados a partir da despesa liquidada em cada ano da subfunção administração direta em saúde. Assim, foi determinado o investimento financeiro/ano, o gasto per capita em saúde, o investimento em APS/ano (disponível desde 2002). Todos os valores foram ajustados conforme o IPCA até o ano de 2013.

O custo anual das ICSAP foi obtido por meio do DATASUS, assim descreveu-se o seu percentual em relação ao investimento financeiro/ano. $\mathrm{O}$ percentual de cobertura populacional pela estratégia de saúde da família (ESF) anual, em Porto Alegre, foi obtido pelas informações fornecidas pelo Departamento de Atenção Básica do Ministério da Saúde (www.dab.saude.gov.br), sendo 
classificada como alta quando atingido cobertura populacional maior que $70 \%$, média entre 50\% e $70 \%$, e baixa quando menor que $50 \%{ }^{15}$.

Inicialmente os dados foram analisados no programa Excel ${ }^{\circledR}$. Foi realizada padronização direta das taxas para retirar a influência das diferenças devidas às estruturas etárias, utilizando-se como referência a população do Estado do Rio Grande do Sul no ano de $2010^{16}$. Posteriormente, com a finalidade de atenuar as variações aleatórias, foram calculadas as médias móveis a cada três anos ${ }^{17}$.

Por meio do Programa Stata ${ }^{\circledR} 11.0$ foi realizada a Regressão de Poisson para analisar a tendência das ICSAP, sendo testada a sobredispersão dos dados com o comando poisgof. O resultado significativo apontou a impropriedade de análise, conduzindo para a Regressão Binomial Negativa. Os resultados foram descritos mediante o coeficiente da razão das médias com seu respectivo intervalo de confiança a $95 \%$ e o resultado do teste $^{18}$.

Realizou-se também a correlação de Spearman, atendendo seus pressupostos, para testar a associação das ICSAP com os investimentos financeiros e os em APS, os gastos per capita em saúde e a cobertura da ESF, sendo considerada como significativa quando alcançou valor superior a 0,50 e valor $p$ menor que $0,05^{19}$.
O projeto de pesquisa foi aprovado pelo Comitê de Ética em Pesquisa da Universidade do Vale do Rio dos Sinos (UNISINOS).

\section{Resultados}

Ao se analisar a tendência das taxas padronizadas das internações por todas as causas, observouse que o maior valor encontrado foi no ano de 2000, com 61,7 internações a cada 1000 habitantes, enquanto o menor foi em 2011 (52,9/1000), correspondendo a uma diminuição de 14,3\%. Posteriormente, realizou-se a análise mediante as médias móveis de três anos, na qual foi possível observar a atenuação das variações aleatórias. A maior taxa foi observada em 2002 (60,4/1000) e a menor em 2009 (54,9/1000), correspondendo a uma diminuição de 10,2\% (Gráfico 1).

Em relação à análise das ICSAP, constatou-se que a maior taxa padronizada foi no ano de 1999 e a menor em 2008, sendo estas, respectivamente, 18,6/1000 e 13,8/1000, variação que representou uma diminuição de $25,8 \%$. Em relação às médias móveis das ICSAP, observaram-se variações muito pequenas, mantendo uma constância das taxas, com a maior no ano de $2001(18,1 / 1000)$ e a menor em 2009 (14,4/1000), o que representou uma queda de 20,5\% (Gráfico 1).

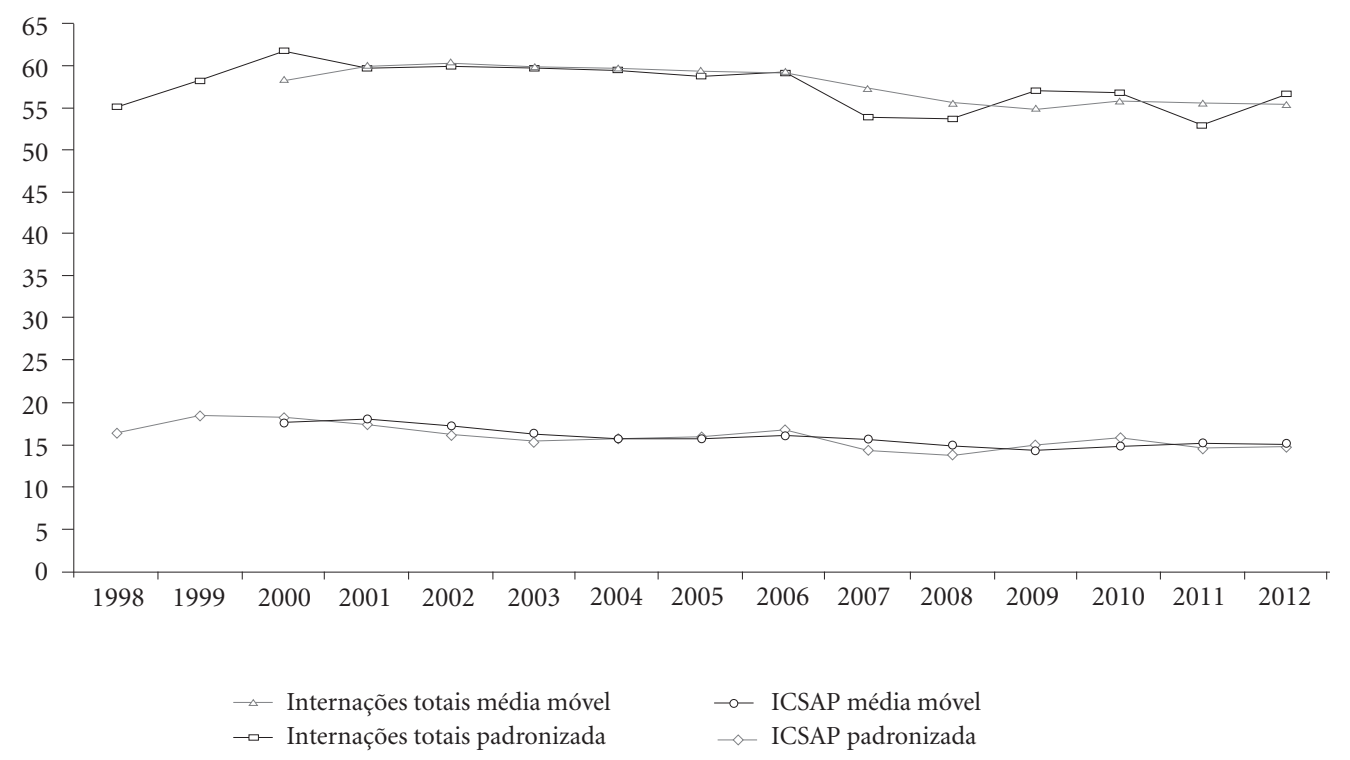

Gráfico 1. Taxa ICSAP e taxa internações por todas as causas com suas respectivas médias móveis. Porto Alegre, 1998-2012. 
No que se refere aos investimentos financeiros realizados em saúde, foi possível observar um pequeno incremento nos valores aplicados. No ano de 1998 foram investidos R\$945.311.379,19, em 2013 os valores atingiram $\mathrm{R} \$ 1.202 .756 .016,06$, representando um aumento de $27 \%$. Comportamento semelhante ocorreu com os gastos per capita em saúde, passando de R\$ 723,89 em 1998 para $\mathrm{R} \$ 819,42$ em 2013, correspondendo a um aumento de $13 \%$ (Tabela 1 ).

Os dados referentes aos investimentos financeiros em APS só estavam disponíveis a partir de 2002, quando foram investidos $\mathrm{R} \$$ 127.910.812,76. Já em 2013, foram investidos R \$ 234.056.900,02. Esta variação correspondeu a um aumento de $83 \%$. Ao relacionar os investimentos em APS com os totais em saúde, a proporção destinada à APS passou de 13,9\% em 2002 para 19,5\% em 2013 (Tabela 1).

Em 1998 os custos das ICSAP atingiram R\$ 26.452.565,18, passando para $\mathrm{R} \$ 27.192 .099,04$ em 2013, o que representou um aumento de apenas 2,8\%. Quando analisados os percentuais dos custos das ICSAP em relação aos investimentos financeiros totais, verificou-se que estes passaram de 2,8\% em 1998 para 2,3\% em 2013 (Tabela 1).

Neste mesmo período, a cobertura populacional pela ESF aumentou cerca de quatro vezes, partindo de $7,7 \%$, chegando a atingir $31,2 \%$ da população em 2013 (Tabela 1), sendo classificada como baixa cobertura.

$\mathrm{Na}$ análise da Regressão de Poisson e da Regressão Binomial Negativa, não foram encontradas diferenças entre as taxas de ICSAP no período estudado, apesar do coeficiente $(0,98)$ mostrar uma redução de cerca de $2 \%$ ao ano, os intervalos de confiança a $95 \%$ variaram de 0,96 a 1,01 e o teste estatístico não apresentou significância $(\mathrm{p}=$ 0,33). Pôde-se perceber que houve um aumento significativo, no período estudado, do investimento financeiro em saúde e da cobertura pela ESF (Tabela 2).

$\mathrm{Na}$ análise da correlação de Spearman foi possível observar que apenas a variável cobertura pela ESF apresentou associação negativa moderada, ou seja, conforme aumentou a cobertura pela ESF, diminuíram as taxas de ICSAP, e essa diferença foi estatisticamente significativa (Tabela 3).

\section{Discussão}

O presente estudo não mostrou alterações nas tendências das taxas de ICSAP no período compreendido entre 1998 e 2012, apesar do aumen-

Tabela 1. Investimento financeiro por ano, gasto per capita em saúde, investimento em atenção primária à saúde, percentual de investimento em atenção primária à saúde em relação ao investimento total em saúde, custo por ICSAP, percentual de custo por ICSAP em relação ao investimento total em saúde e cobertura populacional pela ESF. Porto Alegre, 1998-2012.

\begin{tabular}{|c|c|c|c|c|c|c|c|}
\hline Ano & $\begin{array}{c}\text { Investimento } \\
\text { financeiro/ano (IF) }\end{array}$ & $\begin{array}{c}\text { Gasto per } \\
\text { capita saúde }^{*}\end{array}$ & $\begin{array}{c}\text { Investimento em } \\
\text { APS (IAPS) }\end{array}$ & $\begin{array}{c}\% \\
\text { IAPS/IF }\end{array}$ & $\begin{array}{l}\text { Custo } \\
\text { ICSAP }^{*}\end{array}$ & $\begin{array}{c}\% \text { custo } \\
\text { ICSAP/IF }\end{array}$ & $\begin{array}{c}\text { \% cobertura } \\
\text { ESF }\end{array}$ \\
\hline 1998 & $\mathrm{R} \$ 945.311 .379,19$ & $\mathrm{R} \$ 723,89$ & - & - & $\mathrm{R} \$ 26.452 .565,18$ & 2,8 & 7,7 \\
\hline 1999 & $\mathrm{R} \$ 822.218 .857,53$ & $\mathrm{R} \$ 625,73$ & - & - & $\mathrm{R} \$ 31.993 .787,34$ & 3,9 & 7,7 \\
\hline 2000 & $\mathrm{R} \$ 939.043 .571,46$ & $\mathrm{R} \$ 690,18$ & - & - & $\mathrm{R} \$ 25.110 .603,58$ & 2,7 & 7,6 \\
\hline 2001 & $\mathrm{R} \$ 981.113 .951,74$ & $\mathrm{R} \$ 714,41$ & - & - & $\mathrm{R} \$ 22.746 .500,55$ & 2,3 & 8,8 \\
\hline 2002 & $\mathrm{R} \$ 917.402 .753,32$ & $\mathrm{R} \$ 663,12$ & $\mathrm{R} \$ 127.910 .812,76$ & 13,9 & $\mathrm{R} \$ 19.098 .075,33$ & 2,1 & 14,1 \\
\hline 2003 & $\mathrm{R} \$ 758.341 .965,73$ & $\mathrm{R} \$ 543,96$ & $\mathrm{R} \$ 131.197 .998,37$ & 17,3 & $\mathrm{R} \$ 18.959 .882,90$ & 2,5 & 15,7 \\
\hline 2004 & $\mathrm{R} \$ 757.245 .387,45$ & $\mathrm{R} \$ 539,09$ & $\mathrm{R} \$ 126.996 .040,87$ & 16,8 & $\mathrm{R} \$ 19.946 .068,16$ & 2,6 & 15,3 \\
\hline 2005 & $\mathrm{R} \$ 727.567 .423,37$ & $\mathrm{R} \$ 509,25$ & $\mathrm{R} \$ 139.141 .247,29$ & 19,1 & $\mathrm{R} \$ 19.529 .341,81$ & 2,7 & 20,3 \\
\hline 2006 & $\mathrm{R} \$ 752.376 .439,16$ & $\mathrm{R} \$ 522,14$ & $\mathrm{R} \$ 440.841 .355,82$ & 58,6 & $\mathrm{R} \$ 19.106 .365,18$ & 2,5 & 21,7 \\
\hline 2007 & $\mathrm{R} \$ 849.575 .846,70$ & $\mathrm{R} \$ 584,67$ & $\mathrm{R} \$ 126.598 .095,42$ & 14,9 & $\mathrm{R} \$ 20.056 .431,07$ & 2,4 & 22,0 \\
\hline 2008 & $\mathrm{R} \$ 884.830 .906,85$ & $\mathrm{R} \$ 618,67$ & $\mathrm{R} \$ 129.236 .700,16$ & 14,6 & $\mathrm{R} \$ 22.143 .538,48$ & 2,5 & 22,3 \\
\hline 2009 & $\mathrm{R} \$ 951.334 .421,81$ & $\mathrm{R} \$ 662,43$ & $\mathrm{R} \$ 144.689 .664,44$ & 15,2 & $\mathrm{R} \$ 26.998 .575,15$ & 2,8 & 22,4 \\
\hline 2010 & $\mathrm{R} \$ 983.025 .493,73$ & $\mathrm{R} \$ 697,50$ & $\mathrm{R} \$ 150.463 .812,84$ & 15,3 & $\mathrm{R} \$ 26.678 .722,44$ & 2,7 & 22,8 \\
\hline 2011 & $\mathrm{R} \$ 1.036 .783 .549,14$ & $\mathrm{R} \$ 733,70$ & $\mathrm{R} \$ 180.068 .511,63$ & 17,4 & $\mathrm{R} \$ 25.452 .254,34$ & 2,5 & 23,3 \\
\hline 2012 & $\mathrm{R} \$ 1.220 .210 .942,07$ & $\mathrm{R} \$ 861,29$ & $\mathrm{R} \$ 216.085 .790,78$ & 17,7 & $\mathrm{R} \$ 26.606 .202,93$ & 2,2 & 31,5 \\
\hline 2013 & $\mathrm{R} \$ 1.202 .756 .016,06$ & $\mathrm{R} \$ 819,42$ & $\mathrm{R} \$ 234.056 .900,02$ & 19,5 & $\mathrm{R} \$ 27.192 .099,04$ & 2,3 & 31,2 \\
\hline
\end{tabular}

*Valores corrigidos pelo IPCA até 2013. 
Tabela 2. Regressão Binomial Negativa da taxa padronizada de ICSAP, do investimento financeiro, do investimento em APS, do gasto per capita e da cobertura da ESF, em relação aos anos. Porto Alegre, 1998-2012.

\begin{tabular}{lcccc}
\hline \multicolumn{1}{c}{ Variáveis } & $\begin{array}{c}\text { Razão de } \\
\text { médias }\end{array}$ & IC 95\% & $\mathbf{p}$ \\
\hline ICSAP & 0,98 & 0,96 a 1,01 & $0,3337^{*}$ \\
Investimento financeiro & 1,02 & 1,00 a 1,03 & 0,0238 \\
Investimentos em APS & 1,25 & 0,78 a 2,03 & 0,3239 \\
Gasto per capita & 1,01 & 1,00 a 1,03 & 0,1508 \\
Cobertura ESF & 1,09 & 1,06 a $1,12<$ & $<, 001^{*}$ \\
\hline
\end{tabular}

"Regressão de Poisson

Tabela 3. Correlação de Spearman das taxas padronizadas de ICSAP em relação ao investimento financeiro total em saúde, investimento financeiro em atenção primária à saúde, gasto per capita em saúde e cobertura da ESF. Porto Alegre, 1998-2012.

\begin{tabular}{lcrc}
\hline \multicolumn{1}{c}{ Variáveis } & \multicolumn{1}{c}{$\mathbf{r}$} & \multicolumn{1}{c}{$\mathbf{r}^{2}$} & $\mathbf{p}$ \\
\hline Investimento financeiro & $-0,1964$ & $3,86 \%$ & 0,4829 \\
Investimento em APS & $-0,4595$ & $21,11 \%$ & 0,0849 \\
Gasto per capita & $-0,0071$ & $0,005 \%$ & 0,9798 \\
Cobertura ESF & $-0,7793$ & $60,73 \%$ & 0,0006
\end{tabular}

${ }^{*}$ Regressão de Poisson

to observado nos investimentos financeiros em saúde e na expansão da cobertura da Estratégia Saúde da Família no município de Porto Alegre.

A observação do comportamento das tendências das taxas seguiu uma estratégia de análise baseada na comparação das ICSAP com as internações por todas as causas, na construção de médias móveis e na regressão binomial negativa. Apesar das diminuições percentuais encontradas nas comparações das taxas, a regressão binomial não revelou diferenças significativas no período.

As diversas transformações ocorridas no Sistema Único de Saúde têm provocado diminuição das ICSAP em diferentes locais do Brasil $^{20,21}$, assim esperava-se uma queda das taxas no município de Porto Alegre. Uma das inovações que tem se mostrado relevante na diminuição das ICSAP tem sido a expansão da ESF. Estudos realizados em Minas Gerais mostraram a relação do aumento da cobertura com a diminuição das ICSAP ${ }^{22,23}$. Realizar essa comparação foi o objeti- vo do presente estudo. Deve-se destacar que embora a cobertura por ESF em Porto Alegre tenha aumentado cerca de quatro vezes no período de 15 anos, ela atingiu $31,5 \%$ da população, ficando abaixo dos valores estabelecidos pelo Ministério da Saúde, que tem preconizado uma cobertura de pelo menos 50\% para o estágio de consolidação do Programa Expansão e Consolidação Saúde da Família (PROESF) em municípios entre 500.000 e 2.000.000 de habitantes. Evidentemente que a presente análise não permitiu distinguir a origem dos pacientes que internaram por condições sensíveis, com acesso ou não à ESF, porém mostrou que a cobertura no período estava relacionada com a diminuição das taxas, mas não foi suficiente para impactar o indicador.

Os estudos que encontraram redução das ICSAP relacionadas com a implantação da ESF apresentaram percentuais de cobertura da ESF superiores ao do presente estudo. Em Belo Horizonte, Mendonça et al. ${ }^{22}$ encontraram redução significativa das ICSAP e cobertura de 74,5\% de ESF. E em Montes Claros, MG, Fernandes et al. ${ }^{23}$ encontraram cobertura de ESF ao redor de 50\% e mostraram que quando o controle de saúde era realizado fora da ESF ou quando as hospitalizações eram solicitadas por médicos que não atuavam na ESF, aumentavam em mais de duas vezes a probabilidade de ICSAP.

Esses resultados podem estar associados ao modelo de atenção, que apesar do aumento na cobertura da ESF não impactou no indicador. Seriam necessários mais estudos que avaliassem outras variáveis que poderiam interferir na resolutividade e efetividade da atenção primária, e consequentemente resultar em um impacto na diminuição das ICSAP.

Em relação aos investimentos financeiros foi observado um pequeno incremento no período. Os dados referentes aos gastos per capita mostraram um aumento de $13 \%$ no período de 16 anos. Entretanto, de acordo a com cotação do dólar de cada ano correspondente, verificamos que houve uma diminuição, passando de U\$ 625,77 para U\$ 367,83 . Este valor do final do período ainda foi inferior ao gasto per capita brasileiro, de U\$ 474,00, que tem sido denunciado como insuficiente por diversos autores ${ }^{24,25}$. Além disso, ao se comparar com países latinos americanos, estes valores se encontraram muito abaixo, podese citar como exemplos o gasto per capita de U\$ 851,00 na Argentina, U\$ 825,00 na Costa Rica e U\$ 740,00 no Uruguai, segundo dados de 2013. Isto pode ser explicado pelo fato de que os gastos públicos em saúde no Brasil estão abaixo do 
praticado em outros países desenvolvidos e em desenvolvimento ${ }^{26}$.

Ao se analisar o comportamento dos gastos com APS em Porto Alegre, observou-se um aumento no investimento com o passar dos anos, destacando 2006, que correspondeu à data de implementação do PROESF, quando aproximadamente $59 \%$ do total investido foi destinado à APS. Ao se comparar com os recursos federais, o Brasil destinou $21 \%$ dos gastos totais em saúde para a APS ${ }^{25}$, enquanto Porto Alegre investiu $19,5 \%$ em 2013. Este percentual destinado ficou próximo ao gasto federal e o seu crescimento foi significativo, entretanto, ao se considerar o fato da APS ser a principal porta de entrada ao sistema e responsável por $80 \%$ das demandas de saúde, ficou evidente que os recursos em saúde poderiam ser melhor distribuídos.

Ao verificar os custos das ICSAP, observou-se que estes passaram de $\mathrm{R} \$ 26.452 .565,18 \mathrm{em} 1998$ para R \$ 27.192.099,04 em 2013, não acompanhando o crescimento no investimento financeiro em APS. Não foram encontrados, até o presente momento, estudos em cidades de grande porte que avaliassem os custos com ICSAP. Entretanto, um estudo que avaliou os custos com ICSAP na cidade de São José do Rio Preto, município com cerca de 400 mil habitantes, encontrou um valor de $\mathrm{R} \$ 30.370 .691,08$, nos anos de 2008 a $2010^{13}$. Este valor, correspondente ao somatório dos três anos, está abaixo dos custos de Porto Alegre no ano de 2013. Todavia, não é possível a comparabilidade dos resultados, tendo em vista a diferença do tamanho populacional.

Estudos ecológicos verificando tendências de séries históricas apresentam inúmeras limitações além dos problemas oriundos da utilização de dados secundários (subnotificação e erros de classificação ${ }^{27}$, sofrem com variação aleatória das $\operatorname{taxas}^{17}$ e pela análise agregada não controlam fatores de confusão. Entretanto, recente estudo realizado no Rio Grande do Sul mostrou a adequação do SIH/SUS ${ }^{28}$. Na análise do presente estudo construíram-se médias móveis para "ali- samento da tendência" e padronizaram-se as taxas ajustando o efeito de idade como tentativas de minimizar as limitações.

Além disso, deve-se evidenciar que o delineamento ecológico adotado no presente estudo e sua fonte de dados implicam em limitações evidentes relacionadas ao uso de serviços de saúde tais como número de leitos, tempo de permanência e taxa de ocupação $0^{29} \mathrm{e}$ ainda características individuais demográficas e socioeconômicas como renda familiar e residir em zona rural ${ }^{30}$.

A realização de estudos tendo como fonte de dados o SIH/SUS certamente podem contribuir para seu aprimoramento, desde que utilizados para a avaliação, a gestão e o delineamento de políticas de saúde. No presente estudo não foi possível identificar a origem das ICSAP, principalmente num município organizado em regiões de saúde, o que poderia explicar os resultados encontrados. Ao se pensar que a regionalização é um princípio ordenador dos sistemas de saúde, pode-se apontar a necessidade de incorporação no sistema de informações de alguma variável mais proximal do local de residência dos indivíduos que pudesse identificar seus serviços de saúde de origem.

Por fim, cabe destacar que estudos que avaliam as taxas de ICSAP e suas tendências são importantes fontes de dados para o gerenciamento em saúde e para a formulação de políticas e estratégias que visem à redução destas, de modo a aprimorar o sistema de saúde e os investimentos financeiros aplicados. Ainda ressalta-se que a criação da lista brasileira de ICSAP permitiu a comparabilidade entre estudos nacionais e contribuiu para a sua consolidação como indicador no país. Embora no presente estudo não tenha sido encontrada associação entre a diminuição das taxas de ICSAP com o aumento da cobertura pela ESF e o incremento do investimento financeiro em saúde, há evidências de que estes estejam correlacionados. Recomendam-se outros estudos que avaliem o impacto deste indicador para a reorganização e o aprimoramento do sistema de saúde. 


\section{Colaboradores}

JS Dias da Costa, T Morimoto e JS Arruda participaram da elaboração do projeto, da coleta de dados, da análise dos dados e da redação do artigo. MP Pattussi participou da análise dos dados e da revisão do artigo. GR Bratkowski, M Sopelsa, JS Fritzen, VAF do Canto e MC Marques participaram da análise dos dados e da redação do artigo.

\section{Referências}

1. Alfradique ME, Bonolo PF, Dourado I, Lima-Costa MF, Macinko J, Mendonça CS, Oliveira VB, Sampaio LFR, De Simoni C, Turci MA. Internações por condições sensíveis à atenção primária: a construção da lista brasileira como ferramenta para medir o desempenho do sistema de saúde (Projeto ICSAP - Brasil). Cad Saude Publica 2009; 25(6):1337-1349.

2. Paim J, Travassos C, Almeida C, Bahia L, Macinko J. The Brazilian health system: history, advances, and challenges. Lancet 2011; 377(9779):1778-1797.

3. Campbell SM, Roland MO, Buetow SA. Defining quality of care. Soc Sci Med 2000; 51(11):1611-1625.

4. Starfield B. Atenção primária: equilíbrio entre necessidades de saúde, serviços e tecnologia. Brasília: Unesco; 2002.

5. Shi L, Samuels ME, Pease M, Bailey WP, Corley EH. Patient characteristics associated with hospitalizations for ambulatory care sensitive conditions in South Carolina. South Med J 1999; 92(10):989-998.

6. Billings J, Zeitel L, Lukomnik J, Carey TS, Blank AE, Newman L. Impact of socioeconomic status on hospital use in New York City. Health Aff 1993; 12(1):162173.

7. Nedel FB, Facchini LA, Martín-Mateo M, Vieira LAS, Thumé E. Programa Saúde da Família e condições sensíveis à atenção primária, Bagé (RS). Rev Saude Publica 2008; 42(6):1041-1052.

8. Elias E, Magajewski F. A Atenção Primária à Saúde no sul de Santa Catarina: uma análise das internações por condições sensíveis à atenção ambulatorial, no período de 1999 a 2004. Rev Bras Epidemiol 2008; 11(4):633647.

9. Márquez-Calderón S, Rodríguez del Águila MM, Perea-Milla E, Ortiz J, Bermúdez-Tamayo C. Factores asociados a la hospitalización por procesos sensibles a cuidados ambulatorios en los municipios. Gac Sanit 2003; 17(5):360-367.

10. Mendes Á. A longa batalha pelo financiamento do SUS. Saúde Soc 2013; 22(4):987-990.

11. Mendes EV. O SUS que temos e o SUS que queremos. Rev Min Saúde Pública 2004; 3(4):4-26.

12. Mendes EV. As redes de atenção à saúde. $2^{\mathrm{a}}$ ed. Brasília: Organização Pan-Americana da Saúde; 2011.

13. Ferreira JBB, Borges MJG, Santos LL, Forster AC. Internações por condições sensíveis à atenção primária à saúde em uma região de saúde paulista, 2008 a 2010. Epidemiol Serv Saúde 2014; 23(1):45-56.

14. Dias-da-Costa JS, Borba LG, Pinho MN, Chatkin M. Qualidade da atenção básica mediante internações evitáveis no Sul do Brasil. Cad Saude Publica 2008; 24(7):1699-1707.

15. Costa FF, Calvo MCM. Avaliação da Implantação da Estratégia da Saúde da Família em Santa Catarina em 2004 e 2008. Rev bras Epidemiol 2014; 17(2):557-570.

16. Lilienfeld AM, Lilienfeld DE. Foundations of epidemiology. $2^{\mathrm{a}}$ ed. New York: Oxford University Press; 1980.

17. Latorre MRDO, Cardoso MRA. Análise de séries temporais em epidemiologia: uma introdução sobre os aspectos metodológicos. Rev bras epidemiol 2001; 4(3):145-152.

18. Rosenberg D. Trend analysis and interpretation. Key concepts and methods for maternal and child health professionals. Rockville: Division of Science, Education and Analysis; 1997. 
19. Chen PY, Popovich PM. Correlation: Parametric and Nonparametric Measures. Thousand Oaks: Sage Publication; 2002.

20. Macinko J, Oliveira VB, Turci M a, Guanais FC, Bonolo PF, Lima-Costa MF. The influence of primary care and hospital supply on ambulatory care-sensitive hospitalizations among adults in Brazil, 1999-2007. Am J Public Heal 2011; 101(10):1963-1970.

21. Campos AZ, Theme-filha MM. Internações por condições sensíveis à atenção primária em Campo Grande, Mato Grosso do Sul, Brasil, 2000 a 2009. Cad Saude Publica 2012; 28(5):845-855.

22. Mendonça CS, Harzheim E, Duncan BB, Nunes LN, Leyh W. Trends in hospitalizations for primary care sensitive conditions following the implementation of Family Health Teams in Belo Horizonte, Brazil. Health Policy Plan 2012; 27(4):348-355.

23. Fernandes VBL, Caldeira AP, Faria AA, Neto JFR. Internações sensíveis na atenção primária como indicador de avaliação da Estratégia Saúde da Família. Rev Saude Publica 2009; 43(6):928-936.

24. Santos NR. SUS, política pública de Estado: seu desenvolvimento instituído e instituinte e a busca de saídas. Cien Saude Colet 2013; 18(1):273-280.

25. Capucci P. Financiamento para atenção básica à saúde no Brasil : avanços e desafios. J Manag Prim Heal Care 2014; 5(1):127-128.

26. Mendes EV. 25 anos do Sistema Único de Saúde: resultados e desafios. Estud av 2013; 27(78):27-34.

27. Bittencourt SA, Camacho LAB, Leal M do C. O Sistema de Informação Hospitalar e sua aplicação na saúde coletiva. Cad Saude Publica 2006; 22(1):19-30.

28. Abaid RA. Análise da confiabilidade dos diagnósticos de condições sensíveis à atenção primária nas autorizações de internação hospitalar: um estudo em Santa Cruz do Sul. [dissertação]. Santa Cruz do Sul: Universidade de Santa Cruz do Sul; 2012.

29. Ansari Z, Laditka JN, Laditka SB. Access to health care and hospitalization for ambulatory care sensitive conditions. Med Care Res Rev 2006; 63(6):719-741.

30. Ansari Z, Haider SI, Ansari H, Gooyer T, Sindall C. Patients characteristics associated with hospitalizations for ambulatory care sensitive conditions in Victoria, Australia. BMC Health Services Research 2012; 12:475.

Artigo apresentado em 05/05/2015

Aprovado em 13/08/2015

Versão final apresentada em 15/08/2015 